\title{
Commentary: The effect of modified Pilates based positions on pelvic floor electromyographic (EMG) activity; a pilot study
}

\author{
Mittal Patel ${ }^{1}$ \\ Received: 23 September 2020 / Accepted: 8 October 2020 / Published online: 16 October 2020 \\ (C) The International Urogynecological Association 2020
}

Pelvic floor muscle training has been the cornerstone for conservative management of prolapse and incontinence with various methods suggested for strengthening the pelvic floor muscles. Pilates is gaining popularity as being beneficial for pelvic floor muscles (PFMs) but there is limited and conflicting evidence on its efficacy.

This novel pilot study by Nightingale et al. [1] explores the effect of modified Pilates (MP)-type exercises on pelvic floor muscle (PFM) electromyographic (EMG) activity and whether PFM contraction can be performed during specific MP exercises. EMG feedback was measured via the validated Multiple Array Probe Leiden (MAPLe), which correlates well to individual pelvic floor muscles without interference from the surrounding core muscles. This is different from previous studies using perineometry, ultrasound or patient-reported outcomes alone.

A static exercise programme was undertaken for 20 female volunteers without pelvic floor dysfunction, who were premenopausal, healthy and within BMI range of 18-30. A MAPLe device was used to measure EMG in neutral (lying, standing, kneeling and 'table top'), core (destabilized all fours), teaser (sit-up position with feet lifted from the floor) and plank position (plank on elbows, right and left). EMG readings were taken at rest, during active contraction and Valsalva or straining. The results showed that the EMGs at rest were significantly higher in the core and plank group than in the neutral positions. Higher EMG potentials were achieved in all positions when the volunteers consciously contracted their pelvic floor muscles. During an active contraction, statistically significant higher EMG potentials were noted in both the core and plank groups compared to the neutral group (Mann-Whitney test; core $p=0.0008$, plank $p<0.001$ ).

This is the first study showing that the changes in PFM EMG are higher when MP positions are held for short periods of time, especially when the core is co-contracted, during 'core' and 'plank' positions and during conscious PFM contraction. This proposes that further studies can be done looking specifically at women with pelvic floor dysfunction and longer contractions.

\section{References}

1. Nightingale G, Chandrakumaran K, Phillips C. The effect of modified Pilates-based positions on pelvic floor electromyographic (EMG) activity; a pilot study. Int Urogynecol J. 2020. https://doi. org/10.1007/s00192-020-04529-5.

Publisher's note Springer Nature remains neutral with regard to jurisdictional claims in published maps and institutional affiliations.
Mittal Patel

m.patel6@nhs.net

1 St Mary's Hospital, Imperial College Healthcare NHS Trust, London, UK 